\title{
Conceptual and perceptual information both influence melody identification
}

\author{
MATTHEW D. SCHULKIND \\ Amherst College, Amherst, Massachusetts
}

\begin{abstract}
Three processes have been identified as central to object identification: top-down processing, bottom-up processing, and lateral competition. Six experiments using the perceptual interference paradigm were conducted to assess the relative contributions of these three processes to melody identification. Significant interference was observed only when the target and the distracting information were difficult to distinguish both perceptually and conceptually. Lateral competition-the activation of specific distractor melodies - did not influence the magnitude of interference observed. These results suggest that bottom-up and top-down processes contribute more to melody identification than does lateral competition. The data are discussed in terms of the broader literature on object identification and the relationship between identifying melodies, spoken words, and visual objects.
\end{abstract}

The ability to identify familiar objects has stimulated a great deal of experimental work, which is understandable given the frequency and facility with which this complex task is accomplished in everyday life. However, melodies, which have become an increasingly frequent component of the auditory landscape, have received far less empirical attention than have either spoken words or visual objects. The ability to identify melodies is worthy of increased empirical attention because such identification is so frequently performed. As well, research on melody identification is of theoretical importance because it provides a means for testing the generalizability of theories derived from work with nonmusical stimuli.

In general, theories of object identification have tried to provide answers to two primary questions (Tarr \& Vuong, 2002). What are the fundamental units that are used to identify familiar objects? And how is information in the environment compared with stored representations to allow identification? To answer these questions, theorists have proposed a variety of calculi that draw on three types of mental processes (Jusczyk \& Luce, 2002). Bottom-up processes extract physical information from the stimulus; research has demonstrated that both local (e.g., color or line orientation) and global features (Biederman, 1987) influence visual object identification. Lateral competition refers to competition between exemplars from a given category that interferes with matching a physical stimulus to a stored representation. For example, does the word foreign interfere with the identification of forage? Consensus on this issue has yet to be achieved

This research was supported by a faculty research grant from Amherst College. The author thanks Tamara Rahhal, G. A. Radvansky, and three anonymous reviewers for helpful comments on an earlier draft. Address correspondence to M. D. Schulkind, Department of Psychology, Amherst College, Amherst, MA 01002 (e-mail: mdschulkind@amherst.edu).
(Luce, Pisoni, \& Goldinger, 1990; Marslen-Wilson, 1987). Finally, top-down processes describe how general world knowledge and expectations derived from the experimental situation affect object identification. Identifying a common object is easier if it is presented in a coherent, as opposed to a "jumbled," display (Biederman, Glass, \& Stacy, 1973). As well, similarity ratings of faces are lower if the subjects have previously been instructed to place the faces in different semantic categories (Goldstone, Lippa, \& Shiffrin, 2001). Despite findings such as these, the question of whether conceptual information influences object perception or object labeling remains controversial (Gauthier, James, Curby, \& Tarr, 2002).

Because the melody identification literature has yet to adopt the terminology of the broader field of object identification, a brief description of how bottom-up processes, top-down processes, and lateral competition apply to musical stimuli is warranted. As with visual objects, bottom-up processes in melody identification extract physical characteristics of the stimulus, such as pitch frequency, duration, and frequency spectrum. Melody identification research has focused heavily on bottom-up processing and has shown that listeners are sensitive to manipulations of a wide range of pitch and timing variables (Halpern, 1984; Hébert \& Peretz, 1997; Jones, Summerell, \& Marshburn, 1987; Schulkind, 1999; White, 1960). Listeners use the physical characteristics of the melody to abstract higher order musical properties, such as tonality, meter, and timbre. These higher order properties create a context that influences musical expectations via top-down processes. For example, when asked to determine which of two changes to a melody was larger, adult listeners reliably selected a one-semitone out-ofkey change over a four-semitone change that remained in key (Trainor \& Trehub, 1992). Although not formally studied in the laboratory, extra-musical context might also influence identification. Identifying "Frosty, the 
Snowman" played over a department store loudspeaker is probably easier in December than in mid-July. As well, conceptual knowledge of the to-be-identified stimulus may also influence performance. For example, timbre is more important than pitch when listeners identify very brief (100- or 200-msec) excerpts of popular melodies (Schellenberg, Iverson, \& MacKinnon, 1999). Presumably, the listeners used the timbre of the musical excerpt to infer the correct title (e.g., "I hear a piano, so the song must be $X ")$.

Finally, lateral competition would be observed if one token from the listener's musical database interfered with the identification of another. It would be reasonable to assume that a range of holiday tunes are activated when one enters a store in December and that familiarity with "Rudolph, the Red-Nosed Reindeer" and an understanding that it is a holiday melody would complicate the identification of "Frosty, the Snowman". Although relatively little research has addressed the possibility of lateral competition in melody identification, Hébert and Peretz (1997) found that matching a title to altered versions of familiar melodies was easier when the title was presented early in the trial; this finding did not apply to intact versions of the targets, although ceiling effects $(\approx 95 \%$ correct) clouded interpretation of the data. Schulkind (2002) introduced lateral competition by providing his subjects with an incorrect song title at the start of each trial; this manipulation decreased identification of intact melodies.

The present set of experiments gauged the influences of bottom-up processing, top-down processing, and lateral competition on melody identification, using a classic paradigm in the object identification literature: perceptual interference. Bruner and Potter (1964) demonstrated that initial exposure to a highly degraded version of a target object impaired subsequent identification of that object. Subsequent research has varied both the stimuli used and the method of presentation (Gibson \& Watkins, 1991; Schulkind, 2000, 2002; Snodgrass \& Hirshman, 1991), but most studies have compared performance across two conditions. The fixed condition consists of a single exposure to a moderately degraded version of a target. The ascending condition consists of multiple exposures to the object; starting with a highly degraded version of the object, information is slowly added until the moderately degraded version in the fixed condition is reached. Although the last exposure in the ascending condition is identical to the one exposure in the fixed condition, performance is typically superior in the fixed condition.

The majority of the empirical work in the perceptual interference field has been devoted to investigating the boundary conditions that govern the emergence of the effect (Frederiksen, 1969; Gibson \& Watkins, 1991; Lindfield \& Wingfield, 1998, 1999; Lindfield, Wingfield, \& Bowles, 1994; Luo \& Snodgrass, 1994; Peynircioğlu \& Watkins, 1986; Schulkind, 2000, 2002). Although this research has provided useful information regarding when perceptual interference is or is not observed, a theoretical explanation for the effect has proved elusive. A satisfying explanation for the effect may be lacking because most theories have focused on lateral competition and/or bottom-up processes (Bruner \& Potter, 1964; Snodgrass \& Hirshman, 1991) to the exclusion of other sources of interference (but see, also, Schulkind, 2002). Given the broader literature on object identification, it would seem reasonable to propose that multiple sources of interference combine to produce the effect. Thus, the empirical goal of the present research was to assess the extent to which bottom-up, lateral competition, and top-down processes contribute to the perceptual interference effect in melodic stimuli. The broader theoretical goals of the research were to assess the extent to which these processes influence melody identification and to determine whether parallels exist between the identification of melodies and the identification of other types of stimuli.

\section{EXPERIMENT 1}

All the experiments reported here used musical stimuli and the same basic procedure as in previous work with melodies (Schulkind, 2000, 2002) were used. Every experiment consisted of three conditions (see Figure 1). The fixed condition consisted of a single exposure to the first several notes of a target melody. The ascending condition consisted of multiple exposures; starting with the first two notes, information was slowly incremented on a note-by-note basis until the version heard in the fixed condition was reached. The third condition, which will generally be referred to as the test condition, varied from experiment to experiment. In these critical test conditions, the last exposure was identical to the last exposure in the standard ascending condition and to the one and only exposure in the fixed condition. However, all preceding exposures were replaced by auditory information that was not part of the target but that reproduced selected aspects of the perceptual and/or conceptual structure of the ascending sequence. For example, the first experiment examined the effect of white noise on the magnitude of perceptual interference. White noise was selected as the initial object of study for two reasons. First, it shared none of the conceptual or perceptual features of the to-beidentified stimuli; as such, it provided a good baseline with which to compare the manipulations that followed. Second, white noise provided a means to evaluate a theoretically uninteresting explanation for perceptual interference effects in music. It is possible that any soundregardless of its perceptual and/or conceptual relationship to the target-will interfere with object identification. ${ }^{1}$ According to this hypothesis, the white noise condition should produce as much interference as the ascending condition. However, the failure of white noise to produce significant interference would imply that the interfering stimulus must possess some physical or conceptual relationship to the target. 


\begin{tabular}{|c|c|c|c|c|}
\hline Condition & Experiment & First Exposure & Second Exposure & Third Exposure \\
\hline Fixed & all & \begin{tabular}{l|l|l} 
& & \\
\end{tabular} & & \\
\hline Ascending & all & 2 notes $/ 587 \mathrm{msec}$ & $\frac{}{3 \text { notes } / 883 \mathrm{msec}}$ & 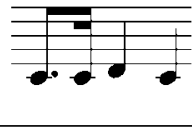 \\
\hline $\begin{array}{l}\text { White } \\
\text { noise }\end{array}$ & 1 & $587 \mathrm{msec}$ of white noise & $883 \mathrm{msec}$ of white noise & $\begin{array}{l}= \\
\rightleftharpoons 0\end{array}$ \\
\hline $\begin{array}{l}\text { Sustained } \\
\text { note }\end{array}$ & 2 & $587 \mathrm{msec}$ of $\mathrm{F}_{4}$ & $883 \mathrm{msec}$ of $\mathrm{F}_{4}$ & 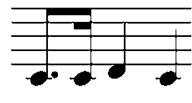 \\
\hline Synthesized & 3 & के & ב) & 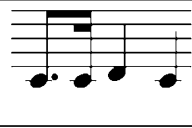 \\
\hline Piano & 4 & $\Longrightarrow$ בP & $\Longrightarrow$ के & 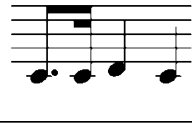 \\
\hline $\begin{array}{l}\text { Constant } \\
\text { distractor }\end{array}$ & 5 & $\Rightarrow 20$ & $\Rightarrow 20$ & \begin{tabular}{l|l} 
\\
$\ldots$
\end{tabular} \\
\hline Switch & 6 & $\overline{\bar{\equiv}}$ & 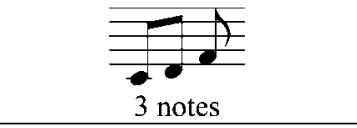 & 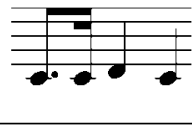 \\
\hline
\end{tabular}

Figure 1. A schematic representation of the experimental conditions used across all six experiments. All music was notated on the treble staff in the key of $\mathbf{F}$ major. The target melody is "Happy Birthday to You," the constant distractor melody was "Old McDonald Had a Farm," and the switch distractor melody was "She'll Be Coming Around the Mountain."

\section{Method}

Subjects. Forty-eight undergraduate volunteers received partial credit toward a course requirement in exchange for their participation and were randomly assigned to one of three counterbalanced orders. None of the subjects in any of the experiments reported here were music majors, nor had any of the subjects participated in any related experiments in this laboratory.

Materials. The 30 songs used in the experiment were selected from a set pretested by a group of 97 undergraduate subjects to ensure their familiarity; a majority of these pilot subjects indicated that they "easily knew most of the words to the song." The songs were drawn from one of the following categories (with a representative tune appearing in parentheses): patriotic ("Yankee Doodle"), Christmas/religious ("Frosty, the Snowman"), pop/folk ("Yesterday"), children's ("Pop Goes the Weasel"), traditional ("Happy Birthday to You"), or movies ("Do Re Me"). A separate group of subjects identified the target stimuli in an ascending sequence in which they were allowed to hear as many as 24 notes; mean percentage correct was $95.5 \%(S D=6.5)$. Thus, the songs were easily identified when the subject population was given sufficient information.

The number of notes heard for each song varied and was selected to yield moderate levels of identification performance, on the basis of previous work (Schulkind, 2000). In the fixed condition, the subjects received one exposure consisting of the selected notes for that song (e.g., the first four notes of "Happy Birthday to You"); only the melody line was played. On average, the songs in the fixed condition consisted of 5.80 notes (range, $4-10 ; S D=1.50$ ) and had an average duration of $2.66 \mathrm{sec}(S D=0.86)$; the average duration per note was approximately $464 \mathrm{msec}(S D=128)$. The lowest note in the corpus was $\mathrm{G}_{3}$, and the highest note was $\mathrm{E}_{5}$, but the vast majority of notes were between $\mathrm{C}_{4}$ and $\mathrm{C}_{5}$. Fifteen of the melodies were presented in the key of $\mathrm{C}$ major, 8 were presented in the key of $\mathrm{F}$ major, 4 were presented in the key of $\mathrm{B} b$ major, and 1 each was presented the keys of G major, D major, and $A b$ major. As well, 9 of the melodies were played in $4 / 4$ meter, 7 in $2 / 4$ meter, and 6 in $3 / 4$ meter; the remaining 8 were played in $6 / 8$ meter.

In the ascending condition, the subjects received multiple exposures to the melody (see Figure 1). The first exposure consisted of the first two notes. Each subsequent exposure added one additional note; that is, the second exposure consisted of the first three notes, the third exposure consisted of the first four notes, and so forth. The final exposure in the ascending condition always contained the same number of notes as the single presentation in the fixed condition. Successive exposures in the ascending condition were separated by $300 \mathrm{msec}$ of silence, because this interval has been shown to yield perceptual interference consistently (Schulkind, 2000).

The white noise condition was constructed using the ascending condition as a base: Each exposure to the melody, save the last, was replaced by a period of white noise equal in duration to selected exposure (see Figure 1). For example, the first two notes (first exposure in the ascending condition) of "Happy Birthday to You" were $587 \mathrm{msec}$ in duration; the first three notes of the song (second exposure in the ascending condition) were $883 \mathrm{msec}$ in duration. In the white noise condition, the first "exposure" to "Happy Birthday 
to You" consisted of $587 \mathrm{msec}$ of white noise; the second "exposure" consisted of $883 \mathrm{msec}$ of white noise. The third exposure was the only one in which the subjects actually heard the melody. The third exposure in the white noise condition consisted of the first four notes of "Happy Birthday to You"; thus, it was identical to the last exposure in the ascending condition and the only exposure in the fixed condition.

The melodies were constructed using a computer software program and were played by a MacIntosh IIsi computer with a piano timbre. The white noise was generated by a different program (SoundEdit) that was also used to splice the melodies and noise. The experimental stimuli were presented by computer and were heard over headphones in a quiet room.

Procedure. The subjects were told that they would be asked to identify familiar songs on the basis of the first several notes. Fixed presentation was explained and illustrated by an example ("Old MacDonald Had a Farm"). The same song was used to illustrate the ascending and the white noise conditions.

Each subject heard each song once in the same random order. Three counterbalanced orders were constructed so that each subject heard 10 songs in each of the three conditions and consecutive songs were never assigned to the same condition. This methodology ensured that, across the experiment, each song was presented an equal number of times in each condition. However, this method did not equate the duration of exposure across conditions; on average, exposure to the target melody was longer in the ascending condition than in the other conditions.

The subjects recorded their responses on an answer sheet. They were instructed to leave the answer sheet blank if they could not guess the title of the song. They were also asked to provide a confidence rating, using the 5-point scale that follows: (1) I have no idea.; (2) It sounds familiar but I am not certain enough to make a guess.; (3) I am not sure but it might be_____ (4) I am pretty sure that it is___ or (5) I am sure that it is___." The five response options were printed at the top of each page of the answer sheet. The subjects were encouraged to guess and were told to write down several song lyrics if they were unsure of the title of the melody. Responses were considered correct if they included either the correct title or at least five consecutive words from the song. For example, "had a very shiny nose" would have been considered a correct response for "Rudolph, the Red-Nosed Reindeer." All other responses were considered incorrect. Because analyses of the confidence rating and the percentage correct data were entirely redundant, only the percentage correct data will be discussed further.

The subjects were given as much time as needed to make their responses. As soon as the experimenter determined that the subjects had completed their response to a song, the next trial was begun. At the end of the experiment, the subjects were given a list of 60 songs: the 30 experimental melodies, plus 30 additional familiar tunes. They were asked to rate the familiarity of each song on a 5-point scale ranging from $1=$ never heard of this song to $5=$ extremely familiar. If a subject claimed to be unfamiliar with a target song, that data point was excluded from all reported analyses. This accounted for less than $1 \%$ of the data in Experiment 1.

\section{Results}

For all the analyses reported in this paper, alpha was set at .05. The pairwise comparisons were all planned; Tukey's honestly significant difference (HSD) was calculated to control the Type I error rate. When appropriate, analyses were conducted using the GreenhouseGeisser adjustment to the degrees of freedom; only the unadjusted values are reported.

Mean percentage correct was averaged over subjects for each presentation style. The best performance was ob- tained in the white noise condition $(M=.66, S E=.03)$, followed by the fixed $(M=.59, S E=.03)$ and the ascending $(M=.50, S E=.03)$ conditions, in that order. An overall one-way analysis of variance (ANOVA) revealed a significant effect of presentation style $[F(2,94)=20.86$, $\left.M S_{\mathrm{e}}=0.015, p<.001\right]$. Pairwise comparisons conducted using Tukey's HSD revealed that all three presentation styles were significantly different from one another.

\section{Discussion}

An overall perceptual interference effect was observed, in that there was a significant difference between performance in the fixed and the ascending conditions. This result matched an often-replicated finding in the literature. However, no interference was observed in the white noise condition; that is, performance in the white noise condition was not significantly worse than performance in the fixed condition. In fact, the white noise condition produced a significant facilitation effect. This result clearly conflicts with the prediction that any sound would produce reliable interference effects and suggests that perceptual interference is not produced by mere auditory stimulation. The interfering stimulus must bear some physical and/or conceptual relationship to the target. The experiments that follow were designed to identify how the target and the interfering stimuli must be related in order to observe interference.

\section{EXPERIMENT 2}

White noise represented a good initial test condition because it shares no features with the musical information contained in the standard ascending sequence. The test conditions in the remaining experiments were designed to vary the physical and conceptual relations to the target melodies. The most obvious difference between white noise and familiar melodies is that the latter are composed of discrete pitches. Therefore, the test condition in Experiment 2 replaced early exposures in the ascending condition with a simple, discrete pitch stimulus: a single, sustained note played in a synthesized timbre. A single sustained note, although consisting of musical material, is not musical; that is, it lacks the organized, coherent modulation of pitch frequency and timing that one associates with melody (Cuddy, 1993). A single sustained note played in a different timbre will activate neither perceptual structures associated with melody identification (bottom-up) nor specific distractor hypotheses (lateral competition). Furthermore, the use of a different timbre will provide conceptual (top-down) information that will allow the listener to segregate the target from the distractor. This analysis suggests that perceptual interference should not obtain in the sustained note condition.

However, the sustained note condition is worth testing because an interference effect in this condition, combined with the failure to observe interference in the white noise condition of Experiment 1, would suggest that interference would be observed if any coherent pitch preceded 
the target. This might be construed as evidence that the observed interference results from the disruption of an auditory processing unit specialized for music. Some researchers have theorized about the possibility of musicspecific modules (Jackendoff, 1987; Peretz \& Morais, 1989; Piccirilli, Sciarma, \& Luzzi, 2000), but a consensus in the literature has not been obtained (see Peretz \& Morais, 1989, for a discussion). Although the failure to observe interference in the sustained note condition would not prove that such a module does not exist, a significant interference effect could be construed as positive evidence.

\section{Method}

Subjects. Sixty undergraduate volunteers received partial credit toward a course requirement in exchange for their participation and were randomly assigned to one of three counterbalanced orders.

Materials. The fixed and ascending conditions were identical to those in the previous experiment. The sustained note condition was similar to the white noise condition in Experiment 1. However, instead of replacing the early exposures in the ascending condition with white noise, the early exposures were replaced by a single sustained note. The pitch of the sustained note was $\mathrm{C}_{5}$ (one octave above middle $\mathrm{C} ; 523.3 \mathrm{~Hz}$ ) and was played with a synthesized timbre similar to an electric keyboard. As such, the timbre of the sustained note (electronic keyboard) and the timbre of the melody (piano) were easily distinguished.

Procedure. The procedure was the same as that in the previous experiment. If a subject claimed to be unfamiliar with a song, that data point was excluded from all the analyses reported. This accounted for less than $1.5 \%$ of the data in Experiment 2.

\section{Results}

Mean percentage correct was averaged over subjects for each presentation style. The best performance was obtained in the sustained note condition $(M=.77, S E=.02)$, followed by the fixed $(M=.74, S E=.02)$, and the ascending $(M=.67, S E=.03)$ conditions. An overall oneway ANOVA revealed a significant effect of presentation style $\left[F(2,118)=8.24, M S_{\mathrm{e}}=0.017, p<.001\right]$. Pairwise comparisons using Tukey's HSD revealed a significant difference between the ascending and the sustained note conditions and between the ascending and the fixed conditions; the difference between the fixed and sustained note conditions did not approach significance.

\section{Discussion}

Significant perceptual interference was observed; performance in the fixed condition was significantly better than performance in the ascending condition. More important, although the sustained note condition did not produce any interference, relative to the fixed condition (in fact, a nonsignificant facilitation effect was observed), performance in the sustained note condition was significantly better than performance in the ascending condition. The failure to observe interference in the sustained note condition suggests that musical material that lacks musical structure is not sufficient to interfere with melody identification. Thus, physical similarity is not enough to create interference; the interfering stimulus must share some features above and beyond being drawn from the same stimulus class. Although obtaining significant in- terference in the sustained note condition would have supported the existence of music-specific modules, the present data do not lead to any strong conclusions regarding this issue.

\section{EXPERIMENTS 3 AND 4}

The results of the first two experiments indicated that neither sound nor "musical sound" was enough to produce perceptual interference. The next step in bringing the test condition closer to the standard ascending sequence was to add musical structure. This was accomplished by replacing the sustained note from Experiment 2 with a simple repeating pattern that instantiated a tonal context. Most Western melodies are organized around a scale (e.g., $\mathrm{C}$ major) that provides a structure or context to help the listener interpret the melody (Krumhansl, 1979, 1990). Even listeners with little or no formal training in music easily recognize erroneous tones that violate the expectations provided by tonal structure (Cuddy, Cohen, \& Miller, 1979).

Experiments 3 and 4 established a tonal context by replacing early exposures in the standard ascending sequence with a repeating pattern consisting of the first, third, and fifth notes in the scale of the target melody. For example, "Happy Birthday to You" was presented in the key of $F$ major, so the repeating sequence consisted of $F, A$, and $\mathrm{C}$. This pattern was selected because previous research had shown that these three notes easily, and unambiguously imply a tonal context (Krumhansl, 1979). ${ }^{2}$ Thus, unlike the sustained note in Experiment 2, the musical pattern in Experiments 3 and 4 invoked a set of musical expectations that should influence subsequent melody perception and identification. The only difference between the two experiments was the timbre of the repeating musical pattern. Whereas Experiment 3 used a synthesized, "electronic" timbre, Experiment 4 used the same piano timbre as the target.

One might expect the instantiation of a musical context to facilitate identification, rather than interfere with it. Although this prediction would follow unambiguously if one were comparing in-key with out-of-key triads, only in-key triads were used in these experiments, because the ascending sequence produces interference even though it invokes the key of the target. Still, interference might be observed if the repeating sequence overprimed the tonic triad, leading to surprise when nontriad notes appeared in the target. However, a timbre change (top-down processing) might allow the listener to overcome the distraction caused by the repeating musical sequence. This analysis predicts that interference would be observed in the piano condition, but not in the synthesized condition.

\section{Method}

Subjects. Fifty-seven undergraduate volunteers participated in Experiment 3; 69 undergraduate volunteers participated in Experiment 4 . All of the subjects received partial credit toward a course requirement in exchange for their participation and were randomly assigned to one of three counterbalanced orders. 
Materials. The fixed and ascending conditions were identical to those used in previous experiments; as such, both were played with a piano timbre. For both the synthesized and the piano conditions, all but the last exposure in the ascending condition was replaced by a repeating sequence of pitches that consisted of the first, third, and fifth degrees of the key in which the piece was written (e.g., $\mathrm{C}_{4}, \mathrm{E}_{4}$, and $\mathrm{G}_{4}$ in $\mathrm{C}$ major). Each tone in the sequences was $375 \mathrm{msec}$ long, and the tones followed one another without interruption. Thus, one full iteration of the sequence lasted $1,125 \mathrm{msec}$. Iterations were pasted into the ascending sequence so that they exactly matched the duration of successive exposures (e.g., the first two notes of "Happy Birthday to You" were replaced with $587 \mathrm{msec}$ of the repeating pattern; see Figure 1). As in the ascending condition, each "exposure" in the synthesized condition was separated by a silent interval of $300 \mathrm{msec}$. The only difference between the two experiments was the timbre in which the repeating sequence was heard: synthesized (Experiment 3) versus piano (Experiment 4).

Procedure. The procedure was the same as that in the previous experiments. If a subject claimed to be unfamiliar with a song, that data point was excluded from all the analyses reported; this accounted for less than $2 \%$ of the data in both experiments.

\section{Results}

Experiment 3. Mean percentage correct was averaged over subjects for each presentation style. The best performance was obtained in the synthesized condition $(M=.67$, $S E=.03)$, followed by the fixed $(M=.63, S E=.03)$ and the ascending $(M=.53, S E=.03)$ conditions. An overall one-way ANOVA revealed a significant effect of presentation style $\left[F(2,112)=13.56, M S_{\mathrm{e}}=0.021, p<.001\right]$. The results of the pairwise comparisons were similar to those obtained in Experiment 2. Tukey's HSD indicated that the fixed and the synthesized conditions did not differ from one another, but both yielded better performance than the ascending condition.

Experiment 4. Mean percentage correct was averaged over subjects for each presentation style. The best performance was obtained in the fixed condition $(M=.73$, $S E=.03)$, followed by the piano $(M=.66, S E=.03)$ and the ascending $(M=.66, S E=.03)$ conditions, which yielded virtually identical levels of performance. An overall one-way ANOVA revealed a significant effect of presentation style $\left[F(2,124)=4.03, M S_{\mathrm{e}}=0.03, p<\right.$ .02]. Pairwise comparisons using Tukey's HSD revealed that the fixed condition yielded significantly better performance than did either the ascending or the piano condition. The piano and the ascending conditions were not significantly different from one another.

\section{Discussion}

Although a standard perceptual interference effect was observed in both experiments (the fixed condition yielded better performance than did the ascending condition), no appreciable difference was observed between the fixed and the synthesized conditions (Experiment 3 ). In contrast, the piano condition (Experiment 4) did yield significant interference. These data have two important implications. First, the interference observed in the piano condition demonstrates that the activation of specific distractor melodies via lateral competition is not required to observe the effect. It should be noted that the interference observed in the piano condition did not arise solely because the subjects were confused about when the target melody began. Were this the case, one would predict a negative correlation between the number of notes in the song and the magnitude of the interference effect. If one assumes that it took a fixed amount of time for the subjects to realize that the target had begun, more notes would be available for the longer songs. However, this correlation was positive and not significantly different from zero $[r(29)=.16, p>.25]$.

Second, the data indicate that the activation of general musical knowledge is not sufficient to produce interference unless the target and the distracting information share a similar timbre. The timbre difference might have eliminated the interference by allowing the target and the distractor to be distinguished via bottom-up processes (i.e., the two sources of information sounded different). Alternatively, the timbre difference could have allowed the listener to segregate the two streams of information on the basis of the source of the stimulus (i.e., the instrument that produced the sound); this would be considered an example of top-down processing.

\section{EXPERIMENT 5}

Experiment 5 was designed to determine whether timbre influenced performance via top-down or bottomup processes. The bottom-up/top-down distinction has several important theoretical implications beyond understanding the particular paradigm used here. If timbre differences eliminate interference primarily via bottomup mechanisms, one could argue that different timbres activate different perceptual units. This would imply that listeners maintain separate mental representation for versions of the same song played by different instruments. This assumption is dubious, given that listeners can easily identify familiar melodies played in an unfamiliar style. For example, it is very unlikely that the subjects in the present experiments had ever heard the target melodies played as they were here - that is, only the melody line with a computerized piano timbre and without vocal accompaniment. However, different instruments are easily distinguished from one another (Robinson \& Patterson, 1995), and timbre has been shown to affect perception (Cusack \& Roberts, 2000; Hartmann \& Johnson, 1991; Iverson, 1995; Pitt, 1995) and recognition memory (Radvansky, Fleming, \& Simmons, 1995), even for highly familiar melodies (Peretz, Gaudreau, \& Bonnel, 1998). Instrumentation has also been shown to affect melody identification, especially for brief excerpts of songs that have been heard only in a single characteristic performance (Schellenberg et al., 1999). Thus, a physical similarity hypothesis cannot easily be dismissed, which leads back to one of the central questions for the present research: What is the root cause of perceptual interference? Is physical similarity the only determinant of the effect, or does conceptual information also play a role? 
An answer to this question was sought by including a condition in which a familiar melody ("Old MacDonald Had a Farm") served as the distracting information; this condition was labeled constant distractor. The distracting melody was played with a piano timbre and in the same general pitch range as the target melodies. Thus, the physical characteristics (bottom-up processing) of the fixed, ascending, and constant distractor conditions were all very similar. If perceptual interference is due primarily to bottom-up processes (e.g., physical similarity), the constant distractor and the ascending conditions should produce similar levels of performance. If, on the other hand, lateral competition contributes to perceptual interference, performance in the constant distractor condition should be lower than that observed in the ascending condition, because the constant distractor condition was composed entirely of perceptual structures associated with a specific nontarget melody (i.e., "Old McDonald"). Finally, if top-down processes influence perceptual interference, performance in the constant distractor condition should be better than performance in the ascending condition. The use of the same distractor melody on each trial in the constant distractor condition provided the subjects with a conceptual cue (the identity of the melody) that would allow them to ignore the distracting information.

\section{Method}

Subjects. Eighty-one undergraduate volunteers received partial credit toward a course requirement in exchange for their participation and were randomly assigned to one of three counterbalanced orders.

Materials. The fixed and the ascending conditions were identical to those used in the previous experiments. The constant distractor condition was created by replacing all but the last exposure in the ascending condition with excerpts of "Old MacDonald Had a Farm" played with a piano timbre. Thus, if the target melody was "Happy Birthday," the first exposure in the constant distractor condition consisted of $587 \mathrm{msec}$ of "Old MacDonald"; this duration was equal to that of the first two notes of "Happy Birthday." The second exposure consisted of $883 \mathrm{msec}$ of "Old MacDonald," and the third and final exposure consisted of the first four notes of "Happy Birthday" (see Figure 1). The subjects were given explicit instructions about trials in the constant distractor condition and were told to identify only the target melody. No subject produced "Old MacDonald" as a response, so the subjects clearly understood the nature of the task.

Procedure. The procedure was the same as that in the previous experiments. If a subject claimed to be unfamiliar with a song, that data point was excluded from all the analyses reported. This accounted for less than $2 \%$ of the data in Experiment 5 .

\section{Results}

Mean percentage correct was averaged over subjects for each presentation style. The best performance was obtained in the fixed condition $(M=.69, S E=.02)$, followed by the constant distractor $(M=.66, S E=.02)$ and the ascending $(M=.62, S E=.02)$ conditions. An overall one-way ANOVA revealed a significant effect of presentation style $\left[F(2,160)=4.38, M S_{\mathrm{e}}=0.024, p<.02\right]$. Pairwise comparisons using Tukey's HSD revealed one significant difference: Performance in the fixed condi- tion differed from performance in the ascending condition. The constant distractor condition did not differ from either the fixed or the ascending condition.

\section{Discussion}

The results in Experiment 5 expand our understanding of the mechanisms that interfere with melody identification. If physical similarity to the target was the only determinant of interference, a significant interference effect should have been observed in the constant distractor condition. Similarly, if lateral competition was the only determinant of interference, a large significant interference effect should have been observed in the constant distractor condition. Neither of these results obtained. Rather, the intermediate level of performance in the constant distractor condition is consistent with the idea that these two forms of interference were balanced by conceptual information (e.g., the identity of the melody) that minimized the effects of the distracting material.

\section{EXPERIMENT 6}

The interpretation of the data from Experiment $5 \mathrm{im}$ plies that removing the conceptual information - the identity of the distracting melody - should reinstate the interference effect. This prediction was tested in Experiment 6 by employing a test condition similar to the constant distractor condition in Experiment 5. In this experiment, however, the identity of the distracting melody varied from song to song and was not revealed to the subject ahead of time. In the switch condition, the subjects might have heard the first two notes of a distractor melody, followed by the first three notes of the distractor, followed by the first four notes of a target melody (see Figure 1). Although the subjects were instructed to expect switch trials at the beginning of the experiment, they were not notified when the switch trials would occur. Therefore, the listeners were unable to ignore the distractor melodies - at least until the switch took place. They were forced to analyze the distractor melodies carefully.

The switch condition in the present experiment and the constant distractor condition in Experiment 5 were similar in that both shared physical features with the target and both introduced lateral competition. The difference is that the constant distractor condition provided the subjects with conceptual information (the identity of the distractor) that distinguished the target from the distractor. If this hypothesis is correct, the switch condition, which did not allow the target and the distractor to be distinguished easily, should reinstate a reliable interference effect.

\section{Method}

Subjects. Fifty-one undergraduate volunteers received partial credit toward a course requirement in exchange for their participation and were randomly assigned to one of three counterbalanced orders.

Materials. The fixed and the ascending conditions were identical to those in the previous experiments. The switch condition was 
created by replacing all but the last exposure in the ascending condition with excerpts from 1 of 10 distractor melodies. The distractor melodies were "Deck the Halls," "For He's a Jolly Good Fellow," "It's a Small World After All," "Jingle Bells," "My Country 'Tis of Thee," "Oh, Susannah," "Ring Around the Rosy," "She'll Be Coming Around the Mountain," "This Land Is Your Land," and "When the Saints Go Marching In." These songs were drawn from a set of stimuli previously tested to be equal in familiarity to the targets (Schulkind, 2002). Unlike the constant distractor condition, the distractor melodies in the switch condition were replaced on a noteby-note basis, rather than on the basis of time. For example, the first two notes of "Happy Birthday to You" were replaced by the first two notes of "She'll Be Coming Around the Mountain," the first three notes of "Happy Birthday to You" were replaced by the first three notes of "She'll Be Coming Around the Mountain," and so on. This procedure was necessary to prevent the subjects from detecting the distractors and also allowed the total number of notes heard in the switch and standard ascending conditions to be equated, something that was not controlled in Experiment 5. The average duration of the stimuli in the ascending condition was 9,831 msec $(S E=829)$; the average duration of the stimuli in the switch condition was $9,790 \mathrm{msec}(S E=977)$. This difference-less than $0.5 \%$ - was not significant $(t<1)$. Thus, the stimuli in the ascending and the switch conditions were equated for both the number of notes and total duration. Three counterbalanced orders were constructed so that each song appeared once in each condition across the experiment. The 10 distractor melodies were randomly assigned to targets in the switch condition. Each distractor was paired with three different targets (one in each counterbalanced order) across the experiment.

Procedure. The procedure was the same as that in the previous experiments. If a subject claimed to be unfamiliar with a song, that data point was excluded from all the analyses reported. This accounted for less than $1 \%$ of the data in Experiment 6 .

\section{Results}

Mean percentage correct was averaged over subjects for each presentation style. The best performance was obtained in the fixed condition $(M=.70, S E=.03)$, followed by the switch $(M=.60, S E=.03)$ and the ascending $(M=$ $.60, S E=.03)$ conditions, which yielded virtually identical performances. An overall one-way ANOVA revealed a significant effect of presentation style $[F(2,100)=9.76$, $\left.M S_{\mathrm{e}}=0.018, p<.001\right]$. Pairwise comparisons using Tukey's HSD indicated that the fixed condition differed from both the ascending and the switch conditions. However, the ascending and the switch conditions did not differ from one another.

\section{Discussion}

Switching the identity of the distracting melody from trial to trial reinstated the interference effect. This occurred because the switch condition, unlike the constant distractor condition, did not provide the subjects with a conceptual cue that allowed them to ignore the distractor. As such, this result reinforces the role that top-down processes play in melody identification. In contrast, the data are inconsistent with a lateral competition explanation that relies on the activation of specific distractors. Although the switch condition activated specific nontarget distractors, it did not produce more interference than the ascending condition. This result does not nullify the possibility that interference can be caused by nonspecific perceptual structures. Finally, the physical similarity of the ascending and the switch conditions (same timbre and pitch range) is consistent with the idea that bottom-up processes contribute to interference.

\section{GENERAL DISCUSSION}

Six experiments assessed the extent to which bottomup, lateral competition, and top-down processes contribute to melody identification in general and to perceptual interference in particular. To review the important results, neither white noise (Experiment 1) nor a single sustained note (Experiment 2) was sufficient to produce interference. Activating general musical structures produced interference, but only if the target and the distractor shared a common timbre (Experiments 3 and 4). Finally, introducing a nontarget melody did not produce interference if the same distractor was used repeatedly (Experiment 5), although interference was observed when the identity of the distractor changed from trial to trial (Experiment 6). Table 1 summarizes the relationship between the target and the distracting information in each experiment. The column labeled "Physical Similarity" indicates whether the distracting information either was drawn from the same pitch range or used the same timbre as the target. "Lateral Competition" indicates whether the distracting information activated either general musical knowledge or the perceptual features of specific distractor melodies. "Conceptual Discrimination" refers to

Table 1

Summary of the Relationship Between the Target and the Ascending and Test Conditions Across All Six Experiments

\begin{tabular}{|c|c|c|c|c|c|}
\hline & \multirow{2}{*}{$\begin{array}{l}\text { Physical } \\
\text { Similarity }\end{array}$} & \multicolumn{2}{|c|}{ Lateral Competition } & \multirow{2}{*}{$\begin{array}{c}\text { Conceptual } \\
\text { Discrimination }\end{array}$} & \multirow[b]{2}{*}{ Interference } \\
\hline & & General & Specific & & \\
\hline Ascending & $\sqrt{ }$ & $\sqrt{ }$ & $\sqrt{ }$ & no & $\sqrt{ }$ \\
\hline White noise & no & no & no & $\sqrt{ }$ & no \\
\hline Held note & $\sqrt{ }$ & no & no & $\sqrt{ }$ & no \\
\hline Musical pattern & $\sqrt{ }$ & $\sqrt{ }$ & no & $\sqrt{ }$ & no \\
\hline Piano & $\sqrt{ }$ & $\sqrt{ }$ & no & no & $\sqrt{ }$ \\
\hline Constant distractor & $\sqrt{ }$ & $\sqrt{ }$ & $\sqrt{ }$ & $\sqrt{ }$ & no \\
\hline Switch & $\sqrt{ }$ & $\sqrt{ }$ & $\sqrt{ }$ & no & $\sqrt{ }$ \\
\hline
\end{tabular}

Note-The interference column indicates whether or not performance in the specified condition was significantly worse than that in the fixed condition. 
whether the listener could easily distinguish the distracting information from the target; in the present experiments, timbre or the identity of the distracting melody was the chief way this was accomplished.

Although the presence of shared physical features may contribute to perceptual interference, it was not sufficient to produce the effect; only two of the six conditions that included shared physical features produced interference. A similar conclusion also applies to the effect of lateral competition. The activation of general musical knowledge did not influence performance (synthesized condition) unless the distracting stimulus shared the same timbre as the target (piano condition). The activation of specific distractor melodies also failed to produce reliable interference; the switch and the ascending conditions did not differ from one another even though the switch condition included nontarget information. As well, the piano and the switch conditions produced comparable levels of interference $(86 \%$ and $90 \%$ of the fixed condition, respectively), even though the piano condition failed to activate specific distractor melodies.

Unlike physical similarity and lateral competition, the ability to distinguish the target and the distractor stimuli conceptually was the only consistent predictor of interference. The piano and the switch conditions were the only test conditions to produce reliable interference, and they were the only conditions in which the target and the distractor were difficult to segregate both conceptually and perceptually. Thus, the data indicate that interference will be observed only if the distractor and the target share both perceptual and conceptual similarities. In other words, the data suggest that both top-down and bottom-up processes contribute to perceptual interference but that lateral competition does not. In light of these data, the name given to the phenomenon ought to be amended to acknowledge the fact that multiple sources of information contribute to the effect. Partial exposure interference is a more apt description of the effect. As well, adopting this terminology would ease confusion created by the use of the term perceptual interference to describe the fact that memory improves when study items are made difficult to perceive (Mulligan, 2000).

The data also suggest that blocking (Snodgrass \& Hirshman, 1991) and competitive activation (Bruner \& Potter, 1960) fail to provide comprehensive accounts for partial exposure interference because they rely too heavily on lateral competition. Although none of the present data was consistent with theories based on lateral competition, these theories cannot be completely dismissed without explaining why effects of lateral competition have been observed on melody identification tasks when the listeners were provided with incorrect song titles (Hébert \& Peretz, 1997; Schulkind, 2002). Although it was not a focus of the present research, some theorists have proposed that recognizing a stimulus and choosing the verbal label for that stimulus are dissociable processes (Gauthier et al., 2002). Stuart and Jones (1995) found a dissociation between environmental sounds and their verbal labels. Environmental sounds were primed by previous exposure to that sound (or a sound generated by the same source), but not by its verbal label; similarly, hearing the sound did not prime the verbal label. Assuming that melody perception and labeling are also dissociable, one could argue that whereas distractor melodies influence perception, distractor titles influence labeling, and that only labeling is susceptible to lateral competition. Clearly, more research would be required to provide empirical support for this hypothesis.

Although the present data suggest that the root causes of partial exposure interference are more complex than most previous theories have suspected, the feature modulation search account (Schulkind, 2002) incorporates both bottom-up and top-down processing. Briefly, the model argues that the key to understanding partial exposure interference is to examine how the distracting information influences the memory search used to identify the target. Perceptual information and bottom-up processes influence the features used to initiate the search, and top-down processes influence the direction of the search. Although the feature modulation search model has the potential to incorporate the present findings into a comprehensive account of the effect, future work will be required to identify the specific processes involved. Enthusiasm for this model is also tempered by the fact that it is the only theory under consideration that was developed using musical stimuli. Whether different modalities should be considered under a single theoretical umbrella is still a topic of debate (Schulkind, 2002). However, the present data do forge a nice link between experiments with music and experiments with verbal material. Peynircioğlu (1990) found that the magnitude of partial exposure interference increased when the subjects were encouraged to try harder. A similar argument could explain why interference was observed in the switch condition, but not in the constant distractor condition. Whereas the former encouraged subjects to try to identify the distractor (because they were unaware that it was a distractor), the latter did not. This suggests that something akin to effort after meaning is required to observe partial exposure interference.

Before examining the implications of these data for object identification in general, two concerns regarding the data and methodology must be addressed. First, one might worry that the interval between exposures in the ascending condition $(300 \mathrm{msec})$ was too small to allow the subjects to segregate successive exposures to the melody. However, although short relative to the average note $(464 \mathrm{msec})$, the $300-\mathrm{msec}$ interval was long relative to the average silent interval. The corpus of melodies included very few rests, so a 300-msec interval was a clear signal that a new exposure was about to begin. As well, during postexperiment interviews, no subject reported any difficulties in parsing the stimuli. Finally, if confusion over the boundaries between successive exposures significantly decreased performance, some interference 
should have been produced by the synthesized condition; none was observed. Therefore, the length of the interstimulus interval cannot explain the consistent differences between the ascending and the fixed conditions.

A second concern was the cross-experiment variability in performance levels in the fixed (range, .59-.73) and ascending (range, .50-.67) conditions. There is no simple explanation for the variability observed here. Given that the subjects were recruited from the same population and in the same manner, it is unlikely that there were significant differences in musical expertise across the different experiments. ${ }^{3}$ However, the magnitude of the interference effect was consistent across experiments $(M=87.6 \%$, $S D=3.2 \%$; range $=84.2-91.8)$ and closely matched the magnitude of the effect observed in the partial exposure effects literature. Across a variety of paradigms and stimulus classes (Gibson \& Watkins, 1991; Luo \& Snodgrass, 1994; Peynircioğlu, 1987, 1990; Peynircioğlu \& Watkins, 1986; Schulkind, 2000, 2002, in press; Snodgrass \& Hirshman, 1991), significant partial exposure interference effects averaged $85 \%$ of baseline $(n=41, S D=5.5)$. Thus, the present data are consistent with past work.

Looking beyond the partial activation interference literature, the present data suggest some commonalities between melody identification and identification of visual objects and spoken words. In particular, the data on lateral competition and top-down processing are central to current debates in the literature. For example, the significant effect of conceptual information on melody identification is consistent with the emerging literature on visual object identification (Gauthier et al., 2002; Goldstone et al., 2001) and with research on the identification of environmental sounds (Ballas, 1993; Ballas \& Mullins, 1991), both of which indicate that conceptual and contextual information influence object identification. This suggests that some components of object identification may operate across different stimulus modalities and classes. In contrast, the failure to observe interference effects from lateral competition conflicts with most current theories of both picture and spoken word identification. The only influential object identification theory that does not incorporate lateral competition is the cohort model of spoken word recognition (Marslen-Wilson, 1987; but see Marslen-Wilson, Moss, \& van Halen, 1996). The link between melody identification and cohort theory could be that the latter places a premium on the sequential processing of information and the features located at the beginning of a word. Melodies are similar in that they are processed sequentially in time and the information at the beginning of the melody appears to be particularly important for identification (Schulkind, in press; Schulkind, Posner, \& Rubin, 2003). However, not every theory of spoken word identification agrees with the cohort theory's emphasis on the initial sounds of a word and its eschewal of lateral competition. How, then, might the failure to observe an effect of lateral competition on this identification task be reconciled with its contribution to spoken word identification and visual object identification? One possibility was discussed before. It could be that lateral competition affects object labeling rather than object perception. In future work, researchers should pay careful attention to the methodology used to introduce lateral competition, with an eye toward separating its effects on these two components of object identification.

In closing, I would like to briefly comment on the benefits of using the partial exposure interference paradigm for the study of object identification. Ideally, an experimental procedure is easily adapted to multiple experimental questions and affords both experimental control and generalizability to the real world. The partial exposure interference paradigm satisfies each of these requirements. It is easily adapted to many questions and many types of stimuli, thus affording the researcher exquisite control of both stimulus presentation and preexperimental knowledge. In addition, identifying an object under conditions of incremental exposure (e.g., by closing the distance between oneself and the object) is a very common experience outside of the laboratory. Therefore, the partial exposure paradigm represents a powerful tool for the study of object identification that heretofore has remained relatively untapped.

\section{REFERENCES}

Ballas, J. A. (1993). Common factors in the identification of an assortment of brief everyday sounds. Journal of Experimental Psychology: Human Perception \& Performance, 19, 250-267.

Ballas, J. A., \& Mullins, R. T. (1991). Effects of context on the identification of everyday sounds. Human Performance, 4, 199-219.

Biederman, I. (1987). Recognition-by-components: A theory of human image understanding. Psychological Review, 94, 115-117.

Biederman, I., Glass, A. L., \& STACY, E. W. (1973). Searching for objects in real-world scenes. Journal of Experimental Psychology, $\mathbf{9 7}$, 22-27.

BrUner, J. S., \& Potter, M. C. (1964). Interference in visual recognition. Science, 144, 424-425.

CudDy, L. L. (1993). Melody comprehension and tonal structure. In T. J. Tighe \& W. J. Dowling (Eds.), Psychology and music: The understanding of melody and rhythm (pp. 19-38). Hillsdale, NJ: Erlbaum.

Cuddy, L. L., Cohen, A. J., \& Miller, J. (1979). Melody recognition: The experimental application of musical rules. Canadian Journal of Psychology, 33, 148-157.

CUSACK, R., \& ROBERTS, B. (2000). Effects of differences in timbre on sequential grouping. Perception \& Psychophysics, 62, 1112-1120.

FREDERIKSEN, J. R. (1969). Response preservation in auditory word recognition. Journal of Experimental Psychology, 79, 48-55.

Gauthier, I., James, T. W., Curby, K. M., \& Tarr, M. J. (2002). The influence of conceptual knowledge on visual discrimination. $\underline{\text { Cogni- }}$ tive Neuropsychology, 20, 507-523.

GiBSON, J. M., \& WATKINS, M. J. (1991). An auditory cue-depreciation effect. American Journal of Psychology, 104, 439-446.

Goldstone, R. L., LIPPA, Y., \& ShIFFrin, R. M. (2001). Altering object representations through category learning. Cognition, 78, 27-43.

Halpern, A. R. (1984). Perception of structure in novel music. $\mathrm{Mem}-$ ory \& Cognition, 12, 163-170.

HARTMANN, W. M., \& JOHNSON, D. (1991). Stream segregation and peripheral channeling. Music Perception, 9, 155-183.

HéBert, S., \& Peretz, I. (1997). Recognition of music in long-term memory: Are melodic and temporal patterns equal partners? Memory \& Cognition, 25, 518-533. 
IVERSON, P. (1995). Auditory stream segregation by musical timbre: Effects of static and dynamic acoustic attributes. Journal of Experimental Psychology: Human Perception \& Performance, 21, 751-763.

JACKENDOFF, R. (1987). Consciousness and the computational mind. Cambridge, MA: MIT Press.

Jones, M. R., Summerell, L., \& Marshburn, E. (1987). Recognizing melodies: A dynamic interpretation. Quarterly Journal of Experimental Psychology, 39A, 89-121.

JusCZYK, P. W., \& Luce, P. A. (2002). Speech perception. In H. Pashler \& S. Yantis (Eds.), Stevens' Handbook of experimental psychology: Vol. 1. Sensation and perception (3rd ed., pp. 493-536). New York: Wiley.

KrumhansL, C. L. (1979). The psychological representation of musical pitch in a tonal context. Cognitive Psychology, 11, 346-374.

KrumHansL, C. L. (1990). Cognitive foundations of musical pitch. New York: Oxford University Press.

LindField, K. C., \& WingField, A. (1998). Perceptual/sensory information versus performance level as indicators of competitive activation in an object identification task: Evidence from aging. Brain \& Cognition, 37, 24-27.

LiNDFIELD, K. C., \& WiNGField, A. (1999). An experimental and computational analysis of age differences in the recognition of fragmented pictures: Inhibitory connections versus speed of processing. Experimental Aging Research, 25, 223-242.

LindField, K. C., WingField, A., \& Bowles, N. L. (1994). Identification of fragmented pictures under ascending versus fixed presentation in young and elderly adults: Evidence for the inhibition-deficit hypothesis. Aging \& Cognition, 1, 282-291.

LuCE, P. A., Pisoni, D. B., \& Goldinger, S. D. (1990). Similarity models of spoken words. In G. Altman (Ed.), Cognitive models of speech perception: Psycholinguistic and computational perspectives (pp. 122147). Cambridge, MA: MIT Press.

Luo, C. R., \& Snodgrass, J. G. (1994). Competitive activation model of perceptual interference in picture and word identification. Journal of Experimental Psychology: Human Perception \& Performance, 20, $50-60$.

MARSLEN-WiLson, W. D. (1987). Functional parallelism in spoken word-recognition. Cognition, 25, 71-102.

Marslen-Wilson W. D., Moss, H. E., \& van Halen, S. (1996). Perceptual distance and competition in lexical access. Journal of Experimental Psychology: Human Perception \& Performance, 22, 13761392.

Mulligan, N. W. (2000). Perceptual interference at encoding enhances item-specific encoding and disrupts relational encoding: Evidence from multiple recall tests. Memory \& Cognition, 28, 539-546.

Peretz, I., Gaudreau, D., \& Bonnel, A. M. (1998). Exposure effects on music preference and recognition. Memory \& Cognition, 26, 884902.

Peretz, I., \& Morais, J. (1989). Music and modularity. Contemporary Music Review, 4, 277-291.

PeynircioĞLU, Z. F. (1987). Inhibition through incremental fragment cuing with primed items. Journal of Experimental Psychology: Learning, Memory, \& Cognition, 13, 569-572.

PEYNIRCIOǦLU, Z. F. (1990). Trying harder may disrupt cue utilization. European Journal of Cognitive Psychology, 2, 363-373.

PeynircioĞLu, Z. F., \& Watkins, M. C. (1986). Cue depreciation: When word fragment completion is undermined by prior exposure to lesser fragments. Journal of Experimental Psychology: Learning, Memory, \& Cognition, 12, 426-431.

PiCCIRILli, M., SCIARMA, T., \& LUZZI, S. (2000). Modularity of music:
Evidence from a case of pure amusia. Journal of Neurology, Neurosurgery, \& Psychiatry, 69, 541-55.

PITT, M. A. (1995). Evidence for a central representation of instrument timbre. Perception \& Psychophysics, 57, 43-55.

Radvansky, G. A., Fleming, K. J., \& SimMONs, J. A. (1995). Timbre reliance in nonmusicians' and musicians' memory for melodies. Music Perception, 13, 127-140.

Robinson, K., \& Patterson, R. D. (1995). The duration required to identify the instrument, the octave, or the pitch chroma of a musical note. Music Perception, 13, 1-15.

Schellenberg, E. G., Iverson, P., \& McKinnon, M. C. (1999). Name that tune: Identifying popular recordings from brief excerpts. Psychonomic Bulletin \& Review, 6, 641-646.

SCHULKIND, M. D. (1999). Long-term memory for temporal structure: Evidence from the identification of well-known and novel songs. Memory \& Cognition, 27, 896-906.

SCHULKIND, M. D. (2000). Perceptual interference decays over short unfilled intervals. Memory \& Cognition, 28, 949-956.

SCHULKIND, M. D. (2002). Feature modulation search: A novel memory search model that extends the perceptual interference effect to musical stimuli. Journal of Experimental Psychology: Learning, Memory, \& Cognition, 28, 346-352.

ScHUlKIND, M. D. (in press). Serial processing in melody identification. Perception \& Psychophysics.

Schulkind, M. D., Posner, R. J., \& Rubin, D. C. (2003). Musical features that facilitate melody identification: How do you know it's "your" song when they finally play it? Music Perception, 21, 217249.

SnODGRass, J. G., \& Hirshman, E. (1991). Theoretical explorations of the Bruner-Potter (1964) interference effect. Journal of Verbal Learning \& Behavior, 30, 273-293.

Stuart, G. P., \& Jones, D. M. (1995). Priming the identification of environmental sounds. Quarterly Journal of Experimental Psychology, 48A, 741-761.

TARR, M. J., \& VUONG, Q. C. (2002). Visual object recognition. In H. Pashler \& S. Yantis (Eds.), Stevens' Handbook of experimental psychology: Vol. 1. Sensation and perception (3rd ed., pp. 287-314). New York: Wiley.

Trainor, L. J., \& Trehub, S. E. (1992). A comparison of infants' and adults' sensitivity to Western musical structure. Journal of Experimental Psychology: Human Perception \& Performance, 18, 394-402.

White, B. W. (1960). Recognition of distorted melodies. American Journal of Psychology, 73, 100-107.

\section{NOTES}

1. One variant on this argument would be to attribute perceptual interference to auditory masking. However, the time course of auditory masking is too short to account for perceptual interference effects (see Schulkind, 2000, for a discussion).

2. Krumhansl (1979) played the three tonic triad notes simultaneously as a chord instead of successively, as was done here.

3. Unfortunately, demographic information on musical experience is not available. However, previous experiments with subjects drawn from the same population (e.g., Schulkind, Posner, \& Rubin, 2003) have shown little variability in musical training.

(Manuscript received May 14, 2003 revision accepted for publication December 16, 2003.) 